\title{
Application of Intravenous Lidocaine in Obese Patients Undergoing Painless Colonoscopy: A Prospective, Randomized, Double-Blind, Controlled Study
}

This article was published in the following Dove Press journal:

Drug Design, Development and Therapy

\author{
Xiaoxiao $\mathrm{Li}^{1, *}$ \\ Xueli Lv' ${ }^{1, *}$ \\ Zhenfei Jiang' \\ Xinrui Nie' \\ Xinghe Wang' \\ Tong $\mathrm{Li}^{\prime}$ \\ Lianyi Zhang ${ }^{2}$ \\ Su Liu (D) ${ }^{1,2}$ \\ 'Jiangsu Province Key Laboratory of \\ Anesthesiology, Xuzhou Medical \\ University, Xuzhou, Jiangsu, People's \\ Republic of China; ${ }^{2}$ Department of \\ Anesthesiology, The Affiliated Hospital of \\ Xuzhou Medical University, Xuzhou, \\ Jiangsu, People's Republic of China \\ *These authors contributed equally to \\ this work
}

\begin{abstract}
Objective: Propofol for procedural sedation and analgesia (PSA) for colonoscopy can result in a high prevalence of severe respiratory depression. Studies have shown that intravenous (IV) infusion of lidocaine can reduce propofol requirements significantly and increase the ventilatory response to carbon dioxide in humans. We tested the hypothesis that IV lidocaine could improve propofol-induced respiratory depression in obese patients during colonoscopy. Methods: Ninety obese patients scheduled for painless colonoscopy were randomized to receive lidocaine $(1.5 \mathrm{mg} / \mathrm{kg}$, then $2 \mathrm{mg} / \mathrm{kg} / \mathrm{h}$, IV) or the same volume of $0.9 \%$ saline. Intraoperative sedation was provided by propofol. The primary outcome was the number of oxygen-desaturation episodes. Secondary outcomes were: the number of apnea episodes; total propofol consumption; time to the first hypoxia episode; time to consciousness loss; intraoperative hemodynamic parameters; awakening time; adverse events; duration of postanesthesia care unit (PACU) stay; satisfaction of endoscopists and patients.
\end{abstract}

Results: Demographic characteristics between the two groups were comparable. The number of oxygen-desaturation episodes in group L $(1.49 \pm 1.12)$ decreased by $0.622(P=0.018)$ compared with that in group $\mathrm{N}(2.11 \pm 1.32)$, and the number of apnea episodes in group L decreased by $0.533(P<0.001)$. Kaplan-Meier curves showed that the median time to the first hypoxia episode was longer in group L (86.78 s) than that in group N (63.83 s) (Log rank $P=0.0008$ ). The total propofol consumption, awakening time, and duration of PACU stay were reduced in group L. There was no significant difference in the prevalence of adverse events $(P>0.05$ for all). Satisfaction scores for endoscopists and patients in group L were higher than that in group $\mathrm{N}(P<0.001)$.

Conclusion: Intravenous infusion of lidocaine could significantly reduce the number of oxygen-desaturation and apnea episodes in obese patients during painless colonoscopy. This method is worthy of clinical promotion.

Clinical Trials Registration: ChiCTR2000028937.

Keywords: lidocaine, propofol, obese patients, colonoscopy

\section{Introduction}

Many gastrointestinal-endoscopy procedures are performed under moderate or deep sedation. Propofol is the most commonly used sedative because of its shorter onset time and faster recovery than that of other anesthetics. However, hypoxemia stemming from propofol sedation continues to be the primary source of morbidity. ${ }^{1}$ As indicated in a large randomized controlled trial, the prevalence of
Correspondence: Su Liu

Department of Anesthesiology,

The Affiliated Hospital of Xuzhou Medical

University, Xuzhou, Jiangsu 221000,

People's Republic of China

Tel +86 I8I I 8309692

$\mathrm{Fax}+86$ 5 I 6-8346-9496

Email liusul I2277@gmail.com 
hypoxemia in patients after intravenous (IV) injection of propofol was $20 \%$ during painless colonoscopy. ${ }^{2}$ Studies have shown that the heavier the bodyweight, the higher the prevalence of hypoxemia. ${ }^{3}$ In addition, the high prevalence of hypoxemia may be associated with mortality, myocardial ischemia, brain injury, and the risk of mechanical ventilation. ${ }^{4-7}$ Extensive researches have been carried out on propofol in combination with other drugs to lower the risk of hypoxemia. For example, dexmedetomidine has been used in combination with propofol to reduce propofol consumption. ${ }^{8}$ Unfortunately, intravenous infusion of dexmedetomidine may be accompanied by hypertension and bradycardia, which will endanger patient safety. ${ }^{9,10}$ The problem of hypoxemia induced by propofol sedation has received more attention recently, but a general agreement in clinical practice is lacking.

Lidocaine is a sodium-channel blocker and is a potential adjunct to propofol sedation. Preclinical studies have shown that IV lidocaine can reduce visceral pain in experimental animals. ${ }^{11}$ Also, lidocaine infusion with a bolus of $0-1.5 \mathrm{mg} / \mathrm{kg}$ followed by $1.5-3 \mathrm{mg} / \mathrm{kg} / \mathrm{h}$ can significantly alleviate abdominal pain in patients. ${ }^{12,13}$ In addition, Labaille et al reported that IV lidocaine could increase the ventilatory response to carbon dioxide in humans. ${ }^{14}$ Forster and colleagues also showed that IV lidocaine could result in a $50 \%$ reduction in propofol requirements when combined with ketamine during procedural sedation and analgesia (PSA) for colonoscopy. ${ }^{15}$ However, whether IV lidocaine can reduce the number of oxygen-desaturation episodes in obese patients undergoing colonoscopy has not been investigated. We hypothesized that IV lidocaine could lower the prevalence of respiratory depression during colonoscopy in obese patients. Therefore, we undertook a prospective, double-blind, randomized controlled trial to explore the efficacy of IV lidocaine on respiratory depression in obese patients undergoing painless colonoscopy.

\section{Materials and Methods}

\section{Ethical Approval of the Study Protocol}

The study protocol was approved by the Ethics Committee of the Affiliated Hospital of Xuzhou Medical University in Xuzhou, China. Written informed consent was obtained from all enrolled participants. The present study complies with the Declaration of Helsinki and adheres to CONSORT guidelines. Before the recruitment of the first patient, our trial was registered on 8 January 2020 on the
Chinese Clinical Trial Registry (ChiCTR2000028937; main researcher: Su Liu). This study was carried out between 13 January and 31 March 2020.

\section{Participants}

Ninety obese patients (American Society of Anesthesiologists (ASA) physical status II-III; 18-75 years; body mass index (BMI) $\geq 30 \mathrm{~kg} / \mathrm{m}^{2}$ ) scheduled to undergo painless colonoscopy in our hospital were enrolled. Then, they were allocated randomly into two groups (45 patients in each group). The exclusion criteria were patients: (i) with II- or III-degree atrioventricular block; (ii) with severe cardiac, pulmonary, hepatic or renal dysfunction; (iii) with diseases of the central nervous system or neuropsychiatric disorders; (iv) using sedativehypnotics or analgesics for $>3$ months; (v) allergic to lidocaine. (vi) refusing to sign informed consent.

\section{Randomization and Masking}

Patients were allocated randomly to the lidocaine group (group L) or normal $(0.9 \%)$ saline group (group $\mathrm{N}$ ) in a $1: 1$ ratio by a computer-generated sequence before colonoscopy. The randomization sequence was retained in an opaque envelope by a nurse. After the patient entered the room, another anesthesiologist who was not involved in postoperative follow-up, data collection or data analyses opened the envelope and prepared the drugs according to the group allocation.

\section{Anesthesia and Intervention}

Patients who met the inclusion criteria were evaluated and screened the day before surgery. The risk of obstructive sleep apnea (OSA) was evaluated using the STOP-Bang questionnaire (comprises eight questions; total score $>5$ indicates a high risk of OSA; 3-4 indicates an intermediate risk of OSA; 0-2 indicates a low risk of OSA). ${ }^{16}$ Preoperative bowel preparation had been completed. An enrolled patient was excluded from the study if: he/she had a cold or fever on the day of the colonoscopy; he/she had pulse oxygen saturation $\left(\mathrm{SpO}_{2}\right)<90 \%$ after entering the room; the duration of colonoscopy lasted 1 hour; endotracheal intubation was indicated.

All patients fasted routinely before surgery without preoperative medication. The patient entered the preparation room $15 \mathrm{~min}$ before the procedure. A peripheral venous channel was established on the right upper limb. Then, $250-300 \mathrm{~mL}$ of sodium lactate Ringer's solution was infused before anesthesia. When the patient entered 
the operating room, electrocardiography, heart rate (HR), blood pressure (BP), $\mathrm{SpO}_{2}$ were monitored continuously. All patients had continuous capnographic monitoring of ventilation activity. The graphic assessment of respiratory activity was provided by the expired carbon dioxide detector attached to the nasal cannula's tip. Oxygen (4 L/min) was supplied via a nasal cannula.

At the beginning of colonoscopy, patients were in the left lateral position. Patients in group L were administered intravenously a bolus dose of $1 \%$ lidocaine $(1.5 \mathrm{mg} / \mathrm{kg})$ before anesthesia induction. Then, $2 \mathrm{mg} / \mathrm{kg}$ of propofol was injected slowly until consciousness was lost. Propofol (4 mg/kg/h) and lidocaine $(2 \mathrm{mg} / \mathrm{kg} / \mathrm{h})$ were infused continuously intraoperatively. Patients in group $\mathrm{N}$ received $0.15 \mathrm{~mL} / \mathrm{kg}$ of physiologic $(0.9 \%)$ saline before anesthesia induction. Then, propofol (2 $\mathrm{mg} / \mathrm{kg}$ ) was injected slowly until consciousness disappeared. Propofol (4 mg/kg/h) and $0.9 \%$ saline $(0.4 \mathrm{~mL} / \mathrm{kg} / \mathrm{h})$ was infused continuously intraoperatively. If necessary, propofol was titrated additionally to produce unconsciousness during anesthesia induction in two groups. Propofol dose was calculated based on the adjusted body weight (ideal body weight + $0.4 \times$ [total body weight-ideal body weight]). ${ }^{17}$ Lidocaine dose was calculated based on the ideal body weight, according to the 2016 Enhanced Recovery After Surgery guidelines for gastrointestinal surgery. ${ }^{18}$ If a polyp or biopsy was taken and the colonoscope was returned to the ileocecum, all infusion drugs were stopped immediately. If patients expressed discomfort (involuntary movement, grimaces), an additional dose of propofol (30-40 mg) was administered. Meanwhile, the rate of propofol infusion was increased by $0.5 \mathrm{mg} / \mathrm{kg} / \mathrm{h}$, and repeated the process if necessary. If the patient suffered from oxygen desaturation and the interference of plethysmographic pulse waveform was excluded by the anesthesiologist, bilateral mandibles were lifted until $\mathrm{SpO}_{2} \geq 95 \%$, and mask ventilation was used to assist breathing if necessary. If $\mathrm{SpO}_{2}$ reverted to normal, the mask was returned to the nasal catheter for oxygen delivery. If hypotension (systolic blood pressure (SBP) $<90 \mathrm{mmHg}$ or descending $20 \%$ basal value) persisted for $\geq 1 \mathrm{~min}$, phenylephrine (40 $\mu \mathrm{g}, \mathrm{IV})$ was administered. If bradycardia (HR $<50 \mathrm{bpm}$ ) occurred, atropine $(0.5 \mathrm{mg}$, IV) was administered. This process was repeated if necessary. Postoperatively, patients were sent to the post-anesthesia care unit (PACU) for further observation.

\section{Outcomes}

The primary outcome was the number of oxygendesaturation episodes (defined as $\mathrm{SpO}_{2}<92 \%$ for $\geq 10 \mathrm{~s}$ ). The main secondary outcomes were: the number of apnea episodes (defined as the absence of respiratory effort for $\geq 10$ $\mathrm{s}$, a flat line for $\geq 10 \mathrm{~s}$ shown on the capnography monitor); ${ }^{19}$ time to the first hypoxia episode (defined as time from induction to first $\mathrm{SpO}_{2}<92 \%$ ); time to consciousness loss (defined as time from induction to the loss of eyelash reflex); total propofol consumption; the hemodynamic parameters, such as HR and SBP, at the following time points: before induction (T0); after anesthesia induction but before the procedure (T1); after endoscope insertion (T2); passing the splenic flexure of the colon (T3); passing the hepatic flexure of the colon (T4); after endoscope removal (T5). Additional secondary endpoints were: awakening time (defined as the time from the end of the procedure to patients answering their name accurately); the sedation level during PACU stay, which was scored using the Ramsay Sedation Scale (1: patients feeling anxious and agitated or restless, or both; 2: patients feeling co-operative, oriented, and tranquil; 3: patients responding to commands only; 4: patients exhibiting brisk response to light tactile stimuli or loud auditory stimulus; 5: patients exhibiting a sluggish response to light tactile stimuli or a loud auditory stimulus; 6: patients exhibiting no response). A score of 2-4 indicated "satisfactory" sedation and a score of 5-6 denoted "excessive" sedation); the pain score $1 \mathrm{~min}$ after awakening (evaluated by a visual analog scale (VAS) from 0 to 10 ; the higher the score, the more intense the pain); the prevalence of adverse events (eg, cardiac dysrhythmias, bradycardia, hypotension, nausea/vomiting, emergence agitation, hypoxia); duration of PACU stay (defined as time from the end of the procedure to discharge from the PACU); the satisfaction of endoscopists and patients (evaluated by a VAS from 0 to 10; the higher the score, the greater the satisfaction). Study outcomes were not altered after patient enrollment.

\section{Statistical Analyses}

Calculation of the sample size was undertaken by PASS 15.0 (NCSS, Kaysville, UT, USA). Based on the results of a pilot study involving 20 patients, the number of oxygendesaturation episodes was $2.2 \pm 1.1$ in group $\mathrm{N}$ and $1.5 \pm 1.1$ in group L. With significance set at 0.05 and power set at $80 \%$, the sample size required to detect differences was 39 patients in each group. Considering a loss to follow-up of $15 \%, 45$ patients were required for each group. 
Numeric variables were analyzed for a normal distribution by the Kolmogorov-Smirnov test. Continuous variables with a normal distribution are expressed as the mean \pm standard deviation (SD) and were compared using the independent-sample $t$-test. Continuous variables with a nonnormal distribution are expressed by the median (interquartile range) and were compared using the Mann-Whitney $U$-test. Categorical variables are presented as numbers (\%) and were compared using the $\chi^{2}$ test or Fisher's exact test. Data on hemodynamic parameters were compared by repeatedmeasure analysis of variance. Kaplan-Meier survival curve analysis with the Log rank test was performed to evaluate the effect of IV lidocaine on the time to first hypoxia episodes. $P<0.05$ was considered significant. Statistical analyses were undertaken using SPSS v24.0 (IBM, Armonk, NY, USA).

\section{Results}

A total of 106 patients were evaluated for study participation. Of these, two patients suffered from severe hypertension, one patient had been using nonsteroidal anti-inflammatory drugs for ankylosing spondylitis over 3 months and eleven patients were excluded for refusing to provide written informed consent. Finally, 92 patients were recruited in our research. After randomization, two patients were excluded; one for having a cold on the day of colonoscopy and the other for having baseline $\mathrm{SpO}_{2}<90 \%$ after hospitalization. Ninety patients (45 in each group) completed colonoscopy (Figure 1). The demographic characteristics between the two groups were comparable (Table 1).

The number of oxygen-desaturation episodes in group L (1.49 \pm 1.12$)$ decreased by 0.622 ( $95 \%$ confidence interval (CI),-1.135 to $-0.110, P=0.018$ ) compared with that in group $\mathrm{N}(2.11 \pm 1.32)$. Compared with group $\mathrm{N}(2.16 \pm 0.37)$, the number of apnea episodes in group L (1.62 \pm 0.49$)$ decreased by 0.533 ( $95 \%$ CI, -0.715 to $-0.352, P<0.001$ ) (Table 2). The intraoperative SBP in group L was lower than that in group $\mathrm{N}$ (Figure 2), whereas the HR in group L was more stable than that in group $\mathrm{N}$.

The Kaplan-Meier curve showed that the median time to the first hypoxia episode was longer in group L ( $86.78 \mathrm{~s}$ ) than that in group $\mathrm{N}(63.83 \mathrm{~s})$ (hazard ratio $=0.430,95 \% \mathrm{CI}, 0.263$ to 0.703 ), and the Log rank test showed that this difference was significant $(P=0.0008)$ (Figure 3$)$. Compared with group $\mathrm{N}(310.73 \pm 30.21 \mathrm{mg})$, the total propofol consumption in

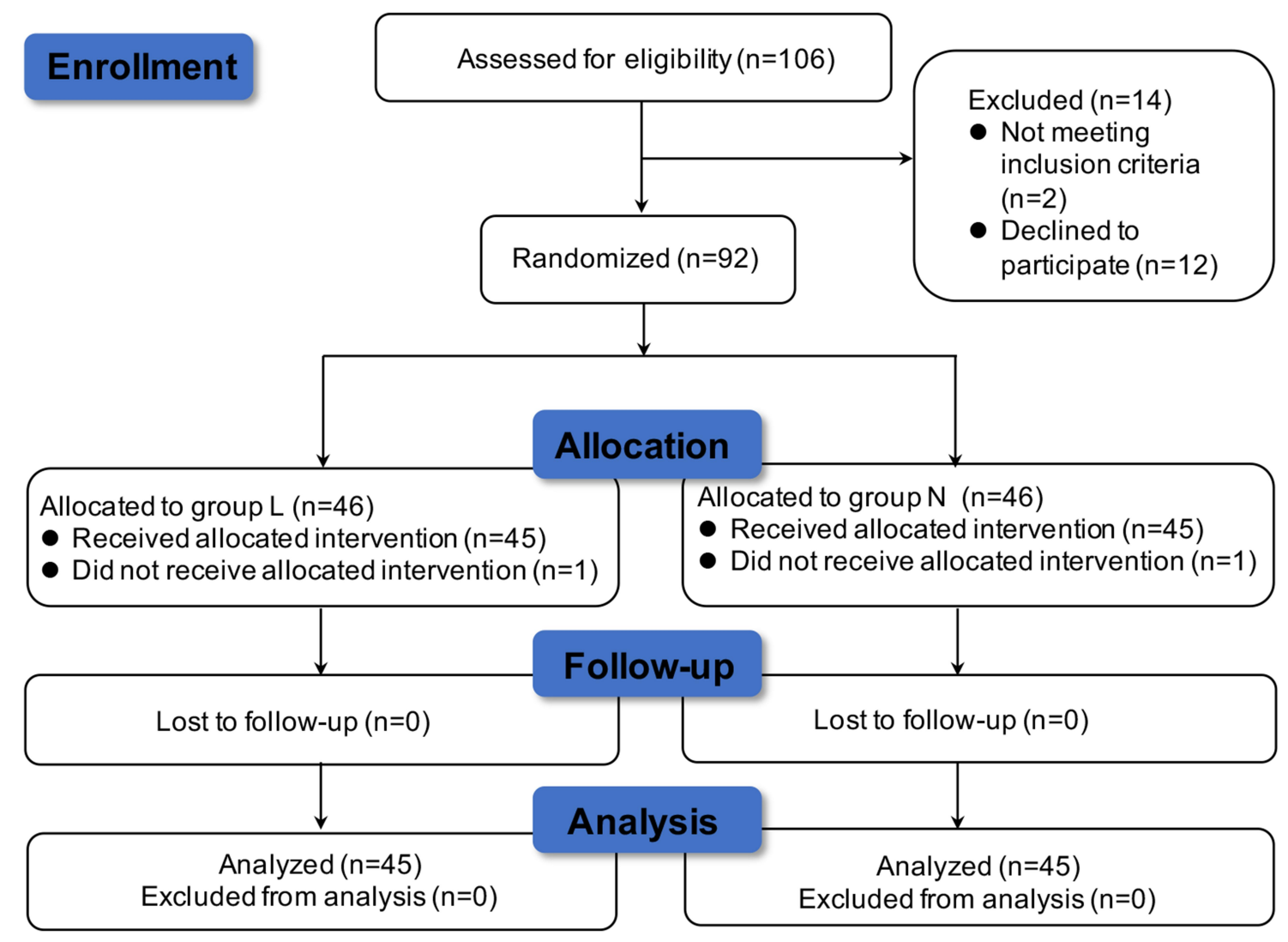

Figure I Study population flow diagram. 
Table I Demographic Profiles at Baseline (Randomization) in Both Groups

\begin{tabular}{|c|c|c|c|}
\hline Variables & $\begin{array}{l}\text { Group } \\
L(n=45)\end{array}$ & $\begin{array}{l}\text { Group } \\
N(n=45)\end{array}$ & P-value \\
\hline Age (years) & $44.38 \pm 7.13$ & $44.93 \pm 7.00$ & 0.710 \\
\hline BMI $\left(\mathrm{kg} / \mathrm{m}^{2}\right)$ & $32.08 \pm 1.72$ & $32.24 \pm 1.48$ & 0.627 \\
\hline $\begin{array}{l}\text { Sex } \\
\qquad \text { Male } \\
\text { Female }\end{array}$ & $\begin{array}{l}30(67) \\
15(33)\end{array}$ & $\begin{array}{l}22(49) \\
23(51)\end{array}$ & 0.088 \\
\hline $\begin{array}{l}\text { ASA physical status } \\
\text { II } \\
\text { III }\end{array}$ & $\begin{array}{l}38(84) \\
7(16)\end{array}$ & $\begin{array}{l}40(89) \\
5(\mathrm{II})\end{array}$ & 0.535 \\
\hline Hypertension & $29(64)$ & $26(58)$ & 0.517 \\
\hline Diabetes & $23(5 \mathrm{I})$ & $28(62)$ & 0.288 \\
\hline $\begin{array}{l}\text { Mallampati score } \\
\text { I } \\
\text { II } \\
\text { III } \\
\text { IV }\end{array}$ & $\begin{array}{l}2(4) \\
9(20) \\
24(53) \\
10(23)\end{array}$ & $\begin{array}{l}1(2) \\
7(16) \\
25(56) \\
12(26)\end{array}$ & 0.427 \\
\hline $\begin{array}{l}\text { Perioperative } \\
\text { Stop-Bang Score }\end{array}$ & $3.96 \pm 1.02$ & $3.96 \pm 1.18$ & 1.000 \\
\hline $\begin{array}{l}\mathrm{SpO}_{2} \text { before Induction } \\
\text { (\%) }\end{array}$ & $98.27 \pm 1.05$ & $97.93 \pm 1.20$ & 0.171 \\
\hline
\end{tabular}

Notes: Data are presented as mean \pm SD or numbers (\%). There were no significant differences among the two groups $(P>0.05)$. Group $L=$ the lidocaine group; Group N= the normal saline group.

Abbreviations: BMI, body mass index; ASA, American Society of Anesthesiologists; $\mathrm{SpO}_{2}$, pulse oxygen saturation.

group L (276.49 $\pm 23.30 \mathrm{mg})$ was reduced by $34 \mathrm{mg}$ (95\% CI, -45.55 to $-22.94, P<0.001)$.

The Ramsay score was lower in group L $(2.84 \pm 0.56)$ than that in group $\mathrm{N}(3.27 \pm 0.58)(P=0.01)$. The 1-min VAS score after colonoscopy was lower in group $\mathrm{L}(2.11 \pm 0.32)$ than that in group $\mathrm{N}(2.80 \pm 0.59)(P<0.001)$. Compared with group $\mathrm{N}$, group L did not show a significant difference in the prevalence of bradycardia, hypotension, nausea/vomiting, or use of atropine and phenylephrine ( $P>0.05$ for all). The satisfaction score for endoscopists and patients in group $\mathrm{L}$ was greater than that in group $\mathrm{N}(P<0.001)$ (Table 3$)$. No cardiac dysrhythmias were detected during the colonoscopy, and no patients had symptoms of toxicity after awakening (eg, dizziness, drowsiness, oral metal odor, mouth paresthesia, blurred vision).

\section{Discussion}

We demonstrated that IV lidocaine during painless colonoscopy in obese patients could reduce the number of oxygen-desaturation and apnea episodes, decrease the total propofol consumption, as well as shorten the duration of consciousness loss, awakening time, and PACU stay compared with propofol for sedation. IV lidocaine could also relieve pain, avoid excessive sedation during recovery, and improve the satisfaction score of endoscopists and patients. Moreover, there were no lidocaine-related adverse reactions.

The popularity of painless endoscopy has relieved the discomfort caused by mechanical stimulation in conventional colonoscopy. Propofol has been the first choice of sedative drug for painless colonoscopy because of its rapid onset of action, strong sedation, short half-life, rapid recovery, and lack of drug accumulation. ${ }^{20,21}$ However, during the induction and maintenance of anesthesia, after intravenous injection of propofol, the blood concentration of propofol increases rapidly, which often results in respiratory depression and low blood pressure. $^{22}$ Furthermore, due to the physiologic changes of the airways that fat infiltration of the upper airway and its surrounding structures, ${ }^{23}$ obese patients are prone to predisposing upper airway narrowing. Also, the volume of distribution of propofol is increased in obese patients

Table 2 Comparisons of Intraoperative Outcomes in Both Groups

\begin{tabular}{|l|l|l|}
\hline Variables & Group L (n=45) & Group N (n=45) \\
\hline Operation duration (min) & $21.65 \pm 3.23$ & $22.95 \pm 4.84$ \\
Total 2\% lidocaine consumption (mg) & $139.89 \pm 4.99$ & $/$ \\
Total propofol consumption (mg) & $276.49 \pm 23.30$ & $310.73 \pm 30.20$ \\
The number of hypoxemia episodes & $1.49 \pm 1.12$ & $2.11 \pm 1.32$ \\
The number of apnea episodes & $1.62 \pm 0.49$ & $2.16 \pm 0.37$ \\
Time to consciousness loss (s) & $26.72 \pm 7.59$ & $36.30 \pm 4.15$ \\
Intraoperative bradycardia & $10(22)$ & $9(20)$ \\
Intraoperative hypotension & $4(9)$ & $<.018$ \\
\hline
\end{tabular}

Notes: Data are presented as mean \pm SD or numbers (\%). Group $L=$ the lidocaine group; Group $N=$ the normal saline group.

Abbreviations: VAS, visual analogue score; PACU, post-anesthesia care unit. 

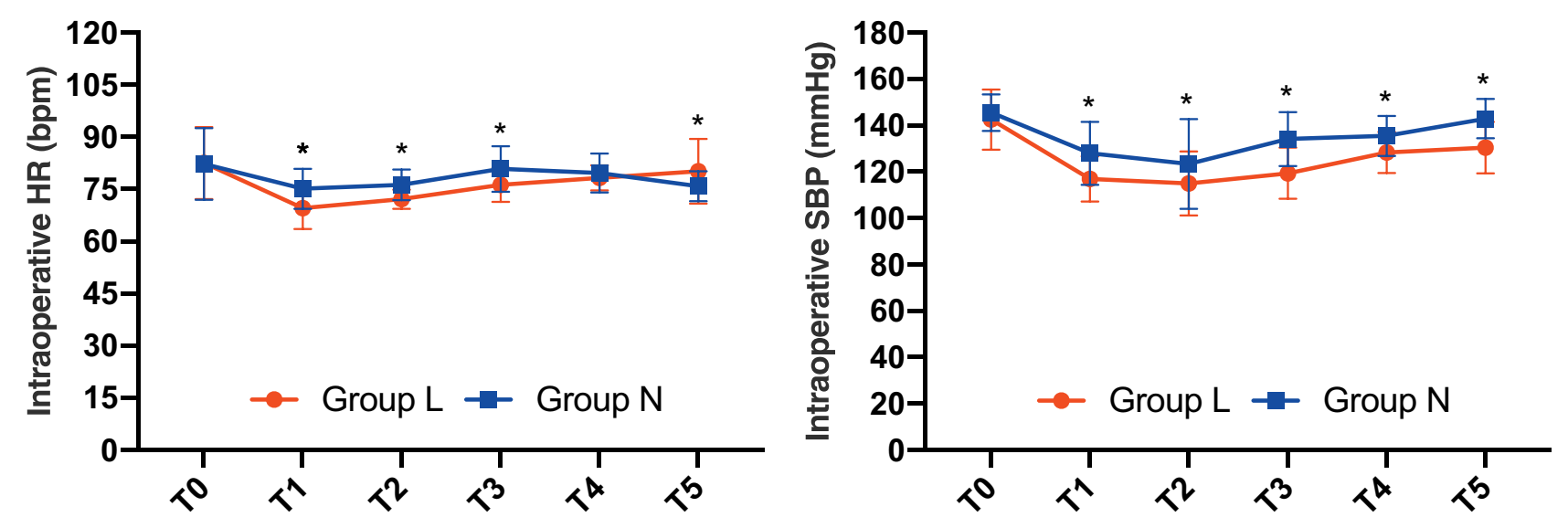

Figure 2 Intraoperative hemodynamic parameters in both groups.

Notes: Group L= the lidocaine group; Group N= The normal saline group. T0= Before induction; $\mathrm{TI}=\mathrm{After}$ anesthesia induction but before the procedure; $\mathrm{T} 2=\mathrm{After}$ endoscope insertion; T3= Passing the splenic flexure of the colon; T4= Passing the hepatic flexure of the colon; T5= After endoscope removal. * P<0.05 Group N vs Group L.

Abbreviations: SBP, systolic blood pressure; HR, heart rate.

for its lipid-soluble characteristics, usually resulting in a higher dose to reach the sedation level and prolonged elimination. ${ }^{24}$ Obese patients have a high prevalence of respiratory depression with propofol for sedation. In severe cases, oxygen desaturation caused by propofol sedation even threatens the safety of patients. ${ }^{25}$ Therefore, it is of crucial clinical value to prevent hypoxemia during painless colonoscopy in obese patients.

There was no significant difference in preoperative BMI or STOP-Bang scores of obese patients between the two groups, which suggests that the risk of suffering respiratory depression in both groups was similar. With reference to previous studies, ${ }^{19,26}$ oxygen desaturation in our study was defined as $\mathrm{SpO}_{2}<92 \%$ for $\geq 10 \mathrm{sec}$, and apnea was defined as an absence of respiratory effort $\geq 10 \mathrm{sec}$. We defined hypoxemia for $\mathrm{SpO} 2<92 \%$ for $\geq 10$ s to exclude the interference of the

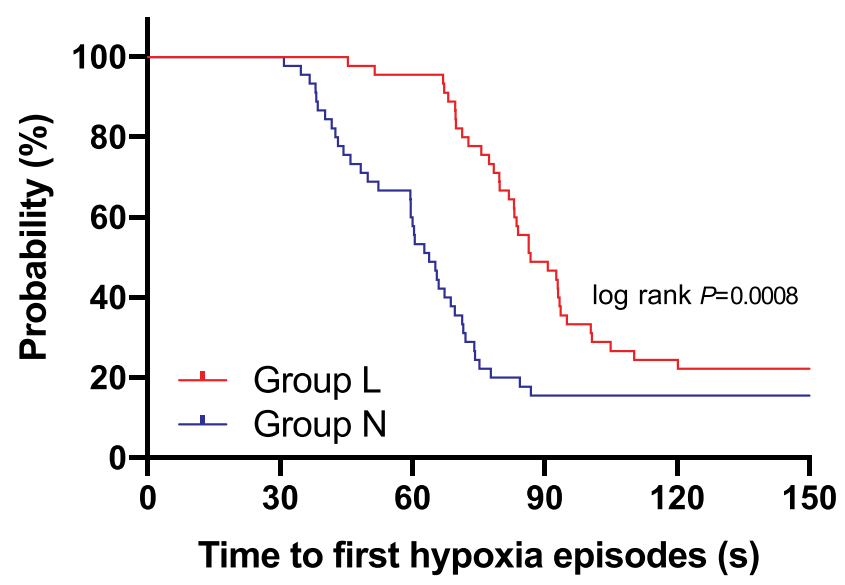

Figure 3 Kaplan-Meier curves for time to first hypoxia episodes. Notes: Group L= the lidocaine group; Group N= the normal saline group. plethysmographic pulse waveform. To avoid the damage caused by prolonged hypoxemia to patients, the observation time was defined as $10 \mathrm{sec}$ instead of a long time. ${ }^{27}$ Compared with group $\mathrm{N}$, the number of oxygen-desaturation episodes and apnea in group L was reduced significantly, and the KaplanMeier curve showed that the median time to the first hypoxia episode was increased by about one-third in group L compared with that in group N. Hence, IV lidocaine could improve respiratory depression in obese patients during painless colonoscopy. A possible explanation for this observation might be

Table 3 Comparisons of Postoperative Outcomes During PACU in Both Groups

\begin{tabular}{|c|c|c|c|}
\hline Variables & $\begin{array}{l}\text { Group } \\
L(n=45)\end{array}$ & $\begin{array}{l}\text { Group } \\
N(n=45)\end{array}$ & P-value \\
\hline $\begin{array}{l}\text { Awakening time } \\
(\min )\end{array}$ & $8.27 \pm 1.30$ & $12.2 \pm 1.95$ & $<0.001$ \\
\hline Bradycardia & $5(\mathrm{II})$ & $7(16)$ & 0.535 \\
\hline Hypotension & $3(7)$ & $5(\mathrm{II})$ & 0.459 \\
\hline Nausea/Vomiting & II (24) & $12(27)$ & 1.000 \\
\hline Emergence agitation & $6(13)$ & $15(33)$ & 0.025 \\
\hline Hypoxia & I (2) & $2(4)$ & 0.557 \\
\hline Ramsay Score & $2.84 \pm 0.56$ & $3.27 \pm 0.58$ & 0.001 \\
\hline $\begin{array}{l}\text { I min VAS after } \\
\text { awakening }\end{array}$ & $2.11 \pm 0.32$ & $2.80 \pm 0.59$ & $<0.001$ \\
\hline $\begin{array}{l}\text { Duration of PACU } \\
\text { stay }\end{array}$ & $14.96 \pm 2.21$ & $22.56 \pm 3.40$ & $<0.001$ \\
\hline $\begin{array}{l}\text { Endoscopists' } \\
\text { satisfaction }\end{array}$ & $8.78 \pm 0.70$ & $8.00 \pm 0.00$ & $<0.001$ \\
\hline Patients' satisfaction & $8.96 \pm 0.37$ & $8.27 \pm 0.62$ & $<0.001$ \\
\hline
\end{tabular}

Notes: Data are presented as mean $\pm S D$ or numbers (\%). Group $L=$ the lidocaine group; Group N= the normal saline group.

Abbreviations: VAS, visual analogue score; PACU, post-anesthesia care unit. 
that, after intravenous administration of lidocaine, the requirement for propofol in obese patients during painless colonoscopy was decreased significantly. Propofol is associated with a higher risk of respiratory depression in a dose-dependent manner. ${ }^{28}$ Hence, the respiratory inhibition caused by propofol was also alleviated significantly. These findings reported by Hans and colleagues suggest that these propofol-sparing effects of IV lidocaine are not mediated by a pure hypnotic effect but rather by anti-nociceptive action, and lidocaine does not affect propofol requirements in the absence of surgical stimulation. $^{29}$ In the study by Altermatt et al, the findings also demonstrate that IV lidocaine can reduce propofol requirements during surgical stimulation and further confirm that the propofol-sparing effects of IV lidocaine are not related to pharmacokinetic interactions between propofol and lidocaine. ${ }^{30}$ Another possible explanation for this finding is that lidocaine does not cause respiratory inhibition and dilates bronchial smooth muscle, ${ }^{31-33}$ thereby preventing airway reactivity on emergence in obese patients. Additionally, IV lidocaine could induce a stimulation of the ventilatory response to carbon dioxide. ${ }^{14}$ Besides the relief of respiratory depression, in group L, the duration of consciousness recovery and duration of PACU stay was shortened by $30 \%$, and the degree of sedation during recovery time was reduced. Therefore, perioperative administration of lidocaine could avoid the oversedation effect caused by an excessive dose of propofol.

Abdominal pain is a common complication of colonoscopy. It is associated with the swelling of the intestinal cavity caused by water and gas injection or mechanical stretching of the intestinal wall caused by the enteroscope during colonoscopy. $^{34}$ The 1-min VAS score during the recovery time in group L was lower than that in group $\mathrm{N}$, which suggests that IV lidocaine produced an analgesic effect. A metaanalysis $^{35}$ involving 354 patients conducted by Li and colleagues found that intravenous infusion of lidocaine $(1.5 \mathrm{mg} / \mathrm{kg} / \mathrm{h})$ inhibited pain after cholecystectomy. Koppert et $\mathrm{al}^{36}$ showed that the analgesic effect of IV lidocaine could be extended to 72 $\mathrm{h}$ after surgery.

Lidocaine is an amide-type local anesthetic. It represses the generation and conduction of action potentials in nerves through inhibition of sodium channels, thereby exerting an analgesic effect at central and peripheral levels. ${ }^{37,38}$ In addition, several studies have shown that IV lidocaine can reduce the release of pro-inflammatory cytokines, alleviate inflammatory pain, and accelerate the recovery of intestinal function. ${ }^{39,40}$ Studies have demonstrated that lidocaine can also block the neurotransmission of damaged nervous tissue, inhibit migration of granulocytes and lysosomes, and reduce production of pro-inflammatory and anti-inflammatory cytokines, thereby exerting an anti-hyperalgesic effect. ${ }^{41,42}$

Although the propofol dose in group L was reduced, the duration of colonoscopy was not prolonged compared with that in group N. This phenomenon did not lower the satisfaction score of endoscopists, suggesting that the reduction of sedative drugs did not affect the endoscopists' procedure. These results are in agreement with those obtained by Forster and colleagues. ${ }^{15}$ We found that, during colonoscopy, the intraoperative HR and SBP in group L were lower than those in group N. This difference can be explained (at least in part) by the analgesic effect provided by lidocaine. Bradycardia and hypotension are common adverse reactions of propofol. However, there was no significant difference between the two groups in the prevalence of bradycardia or hypotension intraoperatively. The reason may be that the sample size was insufficient to reflect the difference between the two groups because the sample calculation was based on the number of hypoxemia episodes.

For patients undergoing continuous intravenous administration of lidocaine during colonoscopy, the main concern was whether the total dose of lidocaine exceeded the safe upper limit that causes neurologic or cardiovascular toxicity. To answer this question, we designed the experiment very carefully. First, in terms of determination of the concentration and dose of lidocaine, we strictly followed the 2016 Enhanced Recovery After Surgery guidelines for gastrointestinal surgery. ${ }^{18}$ It was recommended that lidocaine be injected intravenously at $1.5 \mathrm{mg} / \mathrm{kg}$ within $30 \mathrm{~min}$ before anesthesia induction (based on the ideal body weight) and then infused continuously at $2 \mathrm{mg} / \mathrm{kg} / \mathrm{h}$ (based on the ideal body weight) during the procedure for multimodal analgesia. Second, studies have shown that the safe upper limit of lidocaine concentration in plasma was $5 \mu \mathrm{g} / \mathrm{mL}$, which indicated that a plasma concentration $>5 \mu \mathrm{g} / \mathrm{mL}$ was associated with neurologic symptoms. ${ }^{43,44}$ In the study conducted by Carabalona et al, ${ }^{45}$ 42 obese patients undergoing laparoscopic bariatric surgery were given an IV bolus of lidocaine $1.5 \mathrm{mg} / \mathrm{kg}$ followed by a continuous infusion of $2.0 \mathrm{mg} / \mathrm{kg} / \mathrm{h}$ until the end of the surgery. The median serum concentration was 1.45 (0.98-1.88) $\mu \mathrm{g} / \mathrm{mL}$ and no serum concentrations of lidocaine exceeded 5 $\mu \mathrm{g} / \mathrm{mL}$, which indicates that it is safe to administer intravenously at this infusion rate in obese patients. Compared with non-obese patients, the elimination half-life of lidocaine in obese patients was markedly prolonged, ${ }^{46}$ primarily due to the increase in the absolute volume of distribution induced by higher body weight. ${ }^{47}$ However, there are rare clinical reports of adverse effects of prolonged elimination half-life in obese 
patients. In addition, all patients in group $\mathrm{L}$ were observed closely during the perioperative period, and none of them showed cardiovascular toxicity (eg, increasing intervals, widening QRS complex) or any symptoms of toxicity (eg, dizziness, drowsiness, oral metal odor, mouth paresthesia, blurred vision). Based on previous studies ${ }^{48,49}$ and the clinical signs of patients in our trial, the plasma concentration of lidocaine achieved at this infusion rate was less than the toxic concentration, which is within the safe range of medication.

There were also a few limitations in our study. First, we did not measure the plasma concentration of lidocaine after lidocaine administration. The safety of IV lidocaine was estimated through clinical manifestations of patients. None of the patients showed any signs of local anesthetic intoxication in our study. To a certain extent, it can be considered that the no serum concentration of lidocaine exceeded the non-toxic range. However, the altered physiology of obesity may increase the disposition or alter the clearance of lidocaine, thereby prolonging the elimination half-life. Therefore, future studies should be performed in terms of pharmacokinetics in obese patients to confirm the safety of IV lidocaine for colonoscopy. Second, we did not set a lean control group. However, other studies have shown that IV lidocaine in non-obese patients could result in a remarked reduction of propofol during colonoscopy. ${ }^{15,50}$ There are no lidocaine-related adverse reactions in patients undergoing bariatric surgery. ${ }^{49}$ However, we will further explore the efficacy of intravenous lidocaine in non-obese patients. Third, we did not compare lidocaine with opioids in combination with propofol for procedural sedation and analgesia. Other studies have confirmed the opioid-sparing effect of lidocaine in laparoscopic surgeries. ${ }^{32,51}$ Further studies should be conducted to explore the efficacy of IV lidocaine for procedural sedation and analgesia to decrease opioid consumption and opioid-related complications including respiratory depression. Finally, the recovery of the intestinal function after colonoscopy did not receive attention in our study for the reason that patients discharged from the PACU immediately after recovery, further trials should be confirmed.

\section{Conclusions}

Intravenous infusion of lidocaine could significantly decrease the number of oxygen-desaturation and apnea episodes in obese patients during colonoscopy. This method is worthy of clinical promotion.

\section{Data Sharing Statement}

The de-identified data for individual participants underlying our results can be accessed with approval from the corresponding author 6 months after publication. The study protocol, statistical analyses, and clinical study report will also be available.

\section{Acknowledgments}

The authors thank Dr Zhang for his support in conducting this study and the colleagues and staff of the outpatient operation room and PACU for their cooperation in data collection.

\section{Funding}

This study was supported by the Qing Lan Project of Jiangsu Province, the Nature Science Foundation of Jiangsu Province (BK20161175), "Six One" Project of Jiangsu Province (LGY2016039), Natural Science Research Project of Jiangsu Higher Education Institutions (17KJA3320006). The funds are for Dr. Su Liu. Commercial funding was not received.

\section{Disclosure}

The authors report no conflicts of interest in this work.

\section{References}

1. Goudra B, Singh PM. Critical Analysis of Guidelines for Providing Sedation to Patients Undergoing Gastrointestinal Endoscopy Procedures. Anesth Essays Res. 2019;13(4):601-607. doi:10.4103/aer. AER_135_19

2. Li DN, Zhao GQ, Su ZB. Propofol Target-controlled Infusion in Anesthesia Induction during Painless Gastroscopy. J Coll Physicians Surg Pak. 2019;29:604-607. doi:10.29271/jcpsp.2019.07.604

3. Tsaousi G, Fyntanidou B, Stavrou G, et al. Propofol Sedation for Intragastric Balloon Removal: looking for the Optimal Body Weight Descriptor. Obes Surg. 2019;29(12):3882-3890. doi:10.1007/s11695019-04075-0

4. Abdelmalak BB, Cata JP, Bonilla A, et al. Intraoperative tissue oxygenation and postoperative outcomes after major non-cardiac surgery: an observational study. $B r \quad J$ Anaesth. 2013;110(2):241-249. doi:10.1093/bja/aes378

5. Gill NP, Wright B, Reilly CS. Relationship between hypoxaemic and cardiac ischaemic events in the perioperative period. Br J Anaesth. 1992;68(5):471-473. doi:10.1093/bja/68.5.471

6. Casati A, Fanelli G, Pietropaoli P, et al. Continuous monitoring of cerebral oxygen saturation in elderly patients undergoing major abdominal surgery minimizes brain exposure to potential hypoxia. Anesth Analg. 2005;101 (3):740-747. doi:10.1213/01.ane.0000166974.96219.cd

7. Chiumello D, Brioni M. Severe hypoxemia: which strategy to choose. Crit Care. 2016;20(1):132. doi:10.1186/s13054-016-1304-7

8. Padiyara TV, Bansal S, Jain D, et al. Dexmedetomidine versus propofol at different sedation depths during drug-induced sleep endoscopy: A randomized trial. Laryngoscope. 2020;130(1):257-262. doi:10.1002/lary.27903

9. Edokpolo LU, Mastriano DJ, Serafin J, et al. Discharge Readiness after Propofol with or without Dexmedetomidine for Colonoscopy: A Randomized Controlled Trial. Anesthesiology. 2019;131 (2):279-286. doi:10.1097/ALN.0000000000002809 
10. Amri P, Nahrini S, Hajian-Tilaki K, et al. Analgesic Effect and Hemodynamic Changes Due to Dexmedetomidine Versus Fentanyl During Elective Colonoscopy: A Double-Blind Randomized Clinical Trial. Anesth Pain Med. 2018;8(6):e81077.

11. Ness TJ. Intravenous Lidocaine Inhibits Visceral Nociceptive Reflexes and Spinal Neurons in the Rat. Anesthesiology. 2000;92 (6):1685-1691. doi:10.1097/00000542-200006000-00028

12. Kranke P, Jokinen J, Pace NL, et al. Continuous intravenous perioperative lidocaine infusion for postoperative pain and recovery. Cochrane Database Syst Rev. 2015;7:CD009642.

13. Marret E, Rolin M, Beaussier M, et al. Meta-analysis of intravenous lidocaine and postoperative recovery after abdominal surgery. $\mathrm{Br}$ J Surg. 2008;95(11):1331-1338. doi:10.1002/bjs.6375

14. Labaille T, Clergue F, Samii K, et al. Ventilatory response to $\mathrm{CO} 2$ following intravenous and epidural lidocaine. Anesthesiology. 1985;63(2):179-183. doi:10.1097/00000542-198508000-00011

15. Forster C, Vanhaudenhuyse A, Gast P, et al. Intravenous infusion of lidocaine significantly reduces propofol dose for colonoscopy: a randomised placebo-controlled study. Br J Anaesth. 2018;121 (5):1059-1064. doi:10.1016/j.bja.2018.06.019

16. Chung F, Subramanyam R, Liao P, et al. High STOP-Bang score indicates a high probability of obstructive sleep apnoea. Br J Anaesth. 2012;108(5):768-775. doi:10.1093/bja/aes022

17. Cort 'nez LI, De la Fuente N, Eleveld DJ, et al. Performance of propofol target-controlled infusion models in the obese: pharmacokinetic and pharmacodynamic analysis. Anesth Analg. 2014;119 (2):302-310. doi:10.1213/ANE.0000000000000317

18. Feldheiser A, Aziz O, Baldini G, et al. Enhanced Recovery After Surgery (ERAS) for gastrointestinal surgery, part 2: consensus statement for anaesthesia practice. Acta Anaesthesiol Scand. 2016;60:289-334.

19. Ruehland WR, Rochford PD, O'Donoghue FJ, et al. The new AASM criteria for scoring hypopneas: impact on the apnea hypopnea index. Sleep. 2009;32(2):150-157. doi:10.1093/sleep/32.2.150

20. Smith I, White PF, Nathanson M, et al. An update on its clinical use. Anesthesiology. 1994;81(4):1005. doi:10.1097/00000542-19941000000028

21. Shafer SL. Advances in propofol pharmacokinetics and pharmacodynamics. J Clin Anesth. 1993;5(6):14-21. doi:10.1016/ 0952-8180(93)90003-W

22. Goudra BG, Singh PM. SEDASYS, sedation, and the unknown. $J \quad$ Clin Anesth. 2014;26(4):334-336. doi:10.1016/j. jclinane.2014.02.003

23. Liao YF, Chuang ML, Huang CS, et al. Upper airway and its surrounding structures in obese and nonobese patients with sleep-disordered breathing. Laryngoscope. 2004;114(6):1052-1059. doi:10.1097/00005537-200406000-00018

24. Vargo JJ. Procedural sedation and obesity: waters left uncharted. Gas Trointest Endosc. 2009;70(5):980-984. doi:10.1016/j. gie.2009.07.003

25. Wani S, Azar R, Hovis CE, et al. Obesity as a risk factor for sedation-related complications during propofol-mediated sedation for advanced endoscopic procedures. Gastrointest Endosc. 2011;74 (6):1238-1247. doi:10.1016/j.gie.2011.09.006

26. Writing Committee for the PROBESE Collaborative Group of the PROtective VEntilation Network (PROVEnet) for the Clinical Trial Network of the European Society of Anaesthesiology; Bluth T, Serpa Neto A, Schultz MJ, et al. Effect of Intraoperative High Positive End-Expiratory Pressure (PEEP) With Recruitment Maneuvers vs Low PEEP on Postoperative Pulmonary Complications in Obese Patients: A Randomized Clinical Trial. JAMA. 2019;321 (23):2292-2305. doi:10.1001/jama.2019.7505

27. Kulkas A, Duce B, Leppänen T, Hukins C, Töyräs J. Severity of desaturation events differs between hypopnea and obstructive apnea events and is modulated by their duration in obstructive sleep apnea Sleep Breath. 2017;21(4):829-835. doi:10.1007/s11325-017-1513-6
28. Eastwood PR, Platt PR, Shepherd K, et al. Collapsibility of the upper airway at different concentrations of propofol anesthesia. Anesthesiology. 2005;103(3):470-477. doi:10.1097/00000542-200509000-00007

29. Hans GA, Lauwick SM, Kaba A, et al. Intravenous lidocaine infusion reduces bispectral index-guided requirements of propofol only during surgical stimulation. Br J Anaesth. 2010;105(4):471-479. doi:10.1093/ bja/aeq189

30. Altermatt FR, Bugedo DA, Delfino AE, et al. Evaluation of the effect of intravenous lidocaine on propofol requirements during total intravenous anaesthesia as measured by bispectral index. $\mathrm{Br} J$ Anaesth. 2012;108(6):979-983. doi:10.1093/bja/aes097

31. Rogliani P, Calzetta L, Rendina EA, et al. The influence of propofol, remifentanil and lidocaine on the tone of human bronchial smooth muscle. Pulm Pharmacol Ther. 2013;26(3):325-331. doi:10.1016/j. pupt.2013.01.002

32. De Oliveira GS, Duncan K, Fitzgerald P, et al. Systemic lidocaine to improve quality of recovery after laparoscopic bariatric surgery: a randomized double-blinded placebo-controlled trial. Obes Surg. 2014;24(2):212-218. doi:10.1007/s11695-013-1077-x

33. Riachy M, Khayat G, Ibrahim I, et al. A randomized double-blind controlled trial comparing three sedation regimens during flexible bronchoscopy: dexmedetomidine, alfentanil and lidocaine. Clin Respir J. 2018;12(4):1407-1415. doi:10.1111/crj.12669

34. Karan SB, Bailey PL. Update and review of moderate and deep sedation. Gastrointest Endosc. 2004;14:289.

35. Li J, Wang G, Xu W, et al. Efficacy of intravenous lidocaine on pain relief in patients undergoing laparoscopic cholecystectomy: A meta-analysis from randomized controlled trials. Int $J$ Surg. 2018;50:137-145. doi:10.1016/j.ijsu.2018.01.001

36. Koppert W, Weigand M, Neumann F, et al. Perioperative intravenous lidocaine has preventive effects on postoperative pain and morphine consumption after major abdominal surgery. Anesth Analg. 2004;98:1050-1055. doi:10.1213/01.ANE.0000104582.71710.EE

37. Barletta M, Reed R. Local Anesthetics: pharmacology and Special Preparations. Vet Clin North Am Small Anim Pract. 2019;49 (6):1109-1125. doi:10.1016/j.cvsm.2019.07.004

38. Yardeni IZ, Beilin B, Mayburd E, et al. The effect of perioperative intravenous lidocaine on postoperative pain and immune function. Anesth Analg. 2009;109(5):1464-1469. doi:10.1213/ ANE.0b013e3181bab1bd

39. Xu SQ, Li YH, Wang SB, et al. Effects of intravenous lidocaine, dexmedetomidine and their combination on postoperative pain and bowel function recovery after abdominal hysterectomy. Minerva Anestesiol. 2017;83 (7):685-694. doi:10.23736/S0375-9393.16.11472-5

40. Letson H, Dobson G. Adenosine lidocaine and $\mathrm{Mg}^{2+}$ (ALM) fluid therapy attenuates systemic inflammation, platelet dysfunction and coagulopathy after non-compressible truncal hemorrhage. PLoS One. 2017;12:e0188144. doi:10.1371/journal.pone.0188144

41. Koppert W, Ostermeier N, Sittl R, et al. Low-dose lidocaine reduces secondary hyperalgesia by a central mode of action. Pain. 2000;85 (1):217-224. doi:10.1016/S0304-3959(99)00268-7

42. Herroeder S, Pecher S, Schönherr ME, et al. Systemic lidocaine shortens length of hospital stay after colorectal surgery: a double-blinded, randomized, placebo-controlled trial. Ann Surg. 2007;246(2):192-200. doi:10.1097/SLA.0b013e31805dac11

43. Bromage PR, Robson JG. Concentrations of lignocaine in the blood after intravenous, intramuscular, epidural and endotracheal administration. Anaesthesia. 1961;16(4):4. doi:10.1111/j.1365-2044.1961.tb13426.x

44. Dunn LK, Durieux ME. Perioperative use of intravenous lidocaine. Anesthesiology. 2017;126(4):729-737. doi:10.1097/ ALN.0000000000001527

45. Carabalona JF, Delwarde B, Duclos A, et al. Serum Concentrations of Lidocaine During Bariatric Surgery. Anesth Analg. 2018;130:e5-e9.

46. Abernethy DR, Greenblatt DJ. Lidocaine disposition in obesity. $\mathrm{Am}$ $J$ Cardiol. 1984;53(8):1183-1186. doi:10.1016/0002-9149(84) 90659-3 
47. Beaussier M, Delbos A, Maurice-Szamburski A, et al. Perioperative Use of Intravenous Lidocaine. Drugs. 2018;78(12):1229-1246. doi:10.1007/s40265-018-0955-x

48. Kaba A, Laurent SR, Detroz BJ, et al. Intravenous lidocaine infusion facilitates acute rehabilitation after laparoscopic colectomy. Anesthesiology. 2007;106(1):11-18. doi:10.1097/00000542200701000-00007

49. Plass F, Nicolle C, Zamparini M, et al. Effect of intra-operative intravenous lidocaine on opioid consumption after bariatric surgery: a prospective, randomised, blinded, placebo-controlled study. Anaesthesia. 2020. doi:10.1111/anae.15150
50. Chen M, Lu Y, Liu H, et al. The propofol-sparing effect of intravenous lidocaine in elderly patients undergoing colonoscopy: a randomized, double-blinded, controlled study. BMC Anesthesiol. 2020;20(1):132. doi:10.1186/s12871-020-01049-z

51. Ventham NT, Kennedy ED, Brady RR, et al. Efficacy of intravenous lidocaine for postoperative analgesia following laparoscopic surgery: a meta-analysis. World J Surg. 2015;39(9):2220-2234. doi:10.1007/ s00268-015-3105-6

\section{Publish your work in this journal}

Drug Design, Development and Therapy is an international, peerreviewed open-access journal that spans the spectrum of drug design and development through to clinical applications. Clinical outcomes, patient safety, and programs for the development and effective, safe, and sustained use of medicines are a feature of the journal, which has also been accepted for indexing on PubMed Central. The manuscript management system is completely online and includes a very quick and fair peer-review system, which is all easy to use. Visit http://www. dovepress.com/testimonials.php to read real quotes from published authors. 